\title{
Comparisons of experimental topside electron concentration profiles with IRI and NeQuick models
}

\author{
Pierdavide Coïsson, Sandro Maria Radicella and Bruno Nava \\ Abdus Salam ICTP, Trieste, Italy
}

\begin{abstract}
A critical part of the vertical ionospheric electron concentration profile is the region above its maximum (topside ionosphere) and many attempts have been made to model this region because of the limited experimental data available. Recently, many topside electron concentration profiles obtained with the Intercosmos-19 satellite became accessible through the Internet. The period analyzed corresponds to March 1979 - December 1980, a time interval of high solar activity. The present work describes the comparison of these profiles with the IRI and NeQuick model profiles obtained by driving the models with the values of the maximum electron concentration and its height given by the satellite.
\end{abstract}

Key words topside ionosphere - ionospheric models - electron density profiles

\section{Introduction}

Modeling of the vertical electron concentration profile in the region above the maximum of the ionosphere has not yet reached a satisfactory global representation. One of the reasons is the limited number of data available for that region, since the main sources of such data are satellite topside sounding. In the past decades, ionosondes installed on satellites yielded measurements of topside electron concentration profiles, but only recently have those data sets been made available to the scientific community

Mailing address: Dr. Pierdavide Coïsson, Abdus Salam ICTP, Strada Costiera 11, 34100 Trieste, Italy; e-mail: coissonp@ictp.trieste.it through the WEB. These large databases are useful to compare and validate ionospheric electron concentration models.

In this work we focus attention on two models:

- IRI: the International Reference Ionosphere is a well known empirical model of the ionosphere and widely used (Bilitza, 1990, 1994). For a given location, time and date, IRI describes the electron concentration, electron temperature, ion temperature, and ion composition in the altitude range from about $50 \mathrm{~km}$ to about 2000 $\mathrm{km}$ as well as the Total Electron Content (TEC) to the same altitude. IRI provides monthly medians in the non-auroral ionosphere for magnetically quiet conditions. IRI electron density profiles sources are the coefficients $\left(f_{0} F_{2}\right.$ and $M(3000)$ ) produced by the Radiocommunications Sector of the International Telecommunication Union (ITU-R), incoherent scatter radars (Jicamarca, Arecibo, Millstone Hill, Malvern, St. Santin), the ISIS and Alouette 
topside sounders, and in situ instruments on several satellites and rockets. It can also use experimental values of $F_{2}$ peak electron concentration (i.e. $f_{0} F_{2}$ ) and height as inputs. IRI is updated periodically and has evolved over a number of years.

- NeQuick is a quick-run model for ionospheric applications. It is based on the DGR «profiler» concept (Di Giovanni and Radicella, 1990). It is an Epstein layers description of the electron concentration derived from models developed under European Commission COST 238 and COST 251 actions. The input parameters of the model can be various: ITU-R (former CCIR) coefficients for $f_{0} F_{2}$ and $M(3000) F_{2}$ and $R_{12}$ and/or monthly mean $F 10.7$; measured values of $f_{0} E, f_{0} F_{1}, f_{0} F_{2}$ and $M(3000) F_{2} ; F_{2}$ peak concentration and peak height; regional maps of $f_{0} F_{2}$ and $M(3000) F_{2}$ based on grid values constructed from data obtained at given locations. The output is the vertical or slant profile of electron density and the corresponding total electron content to any given height up to 20000 km (Hochegger et al., 2000; Radicella and Leitinger, 2001).

\section{Comparison technique}

The database used in this work is the IZMIRAN database of topside profiles accessible through the web (http://antares.izmiran.rssi.ru/ projects/IK19/), which at the time of presentation of this work contained more than 5000 topside electron concentration profiles from the Intercosmos-19 satellite, in the period of high solar activity from March 1979 to December 1980 (fig. 1). In that period $R_{12}$, twelve-month smoothed relative sunspot number, was between 136.5 and 164.5; the maximum occurred in December 1979. The height range of the profiles goes from the electron concentration maximum in the $F_{2}$ region up to $500-1000 \mathrm{~km}$, this range is determined by the elliptic orbit of the satellite with a perigee of $502 \mathrm{~km}$ and an apogee of 995 $\mathrm{km}$. All profiles considered reached the $F_{2}$ peak, being able to determine in all cases $h_{m} F_{2}$ and $N_{m} F_{2}$, used to drive the models.

The IRI and NeQuick electron concentration profiles were calculated driving the models with: peak parameters $h_{m} F_{2}$ and $N_{m} F_{2}$ derived from the topside profiles, $R_{12}$, geographic coordinates and time at which the experimental profile was taken.

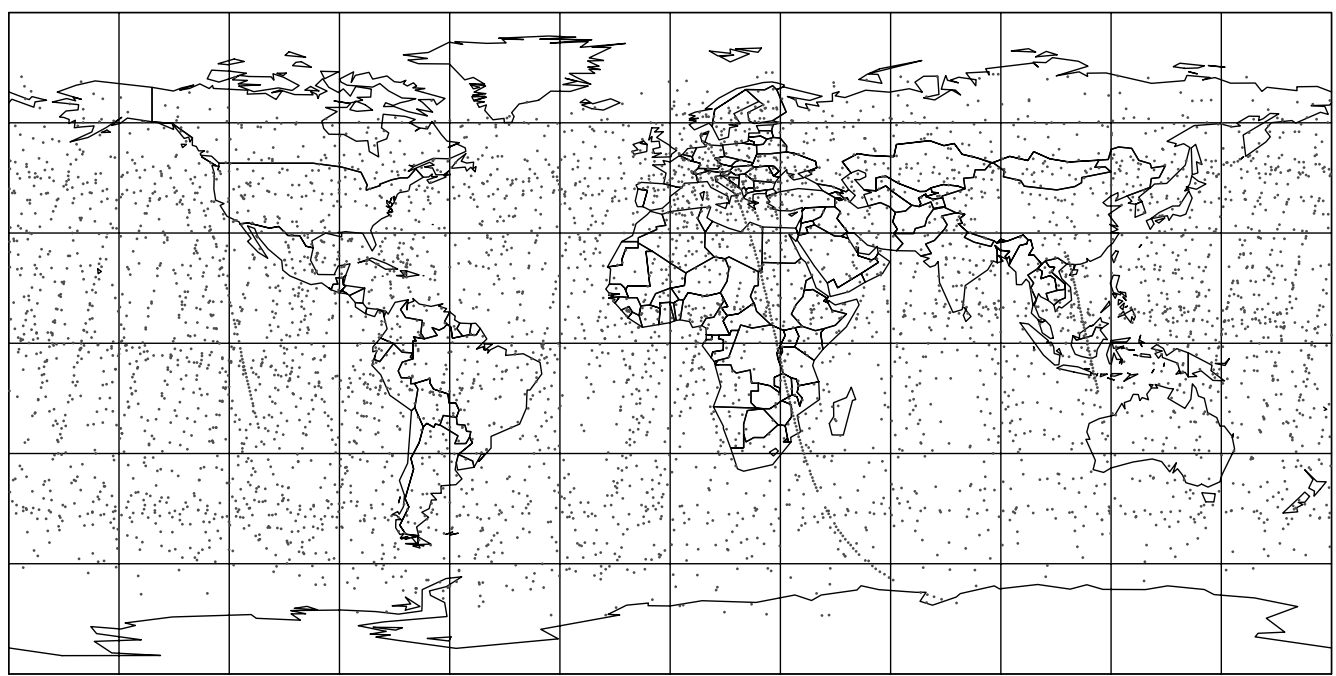

Fig. 1. Geographic location of the analyzed profiles March 1979 - December 1980. 


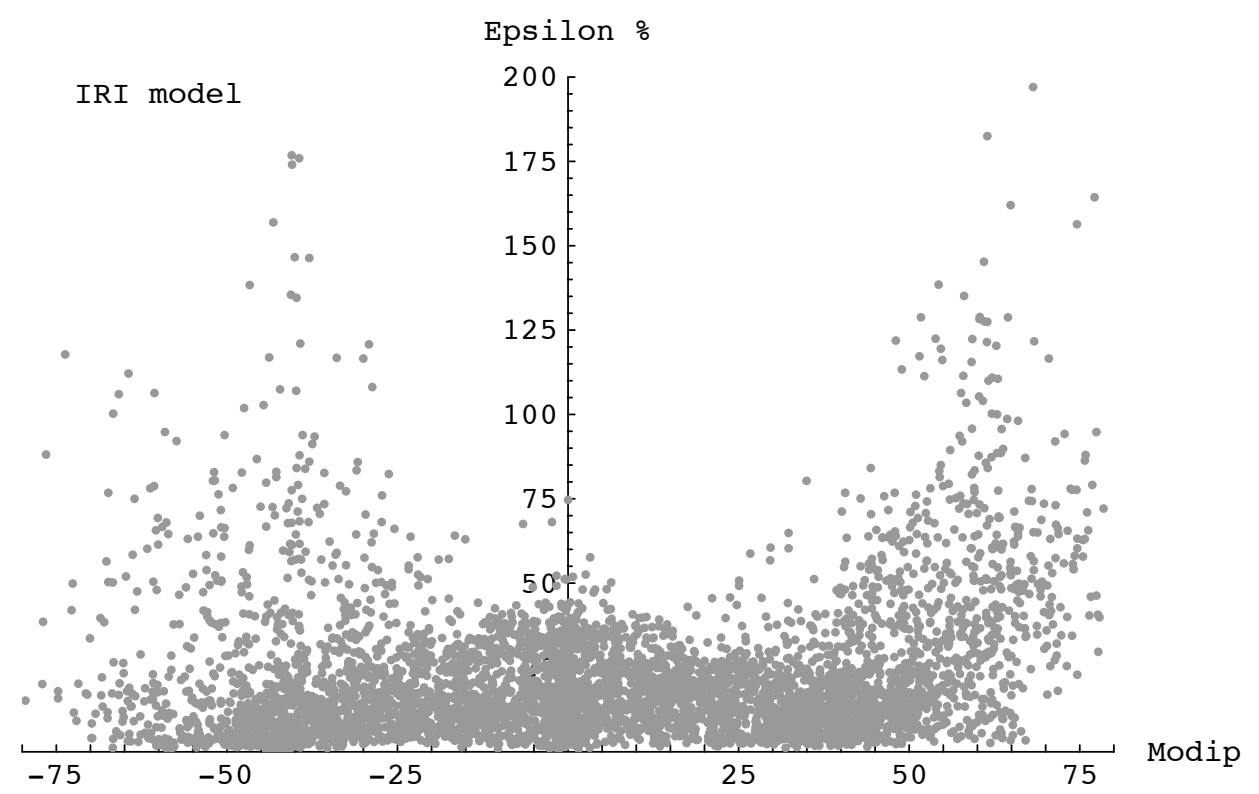

Fig. 2. Scatter plot of $\varepsilon$ computed for IRI model profiles as function of Modified Dip Latitude.

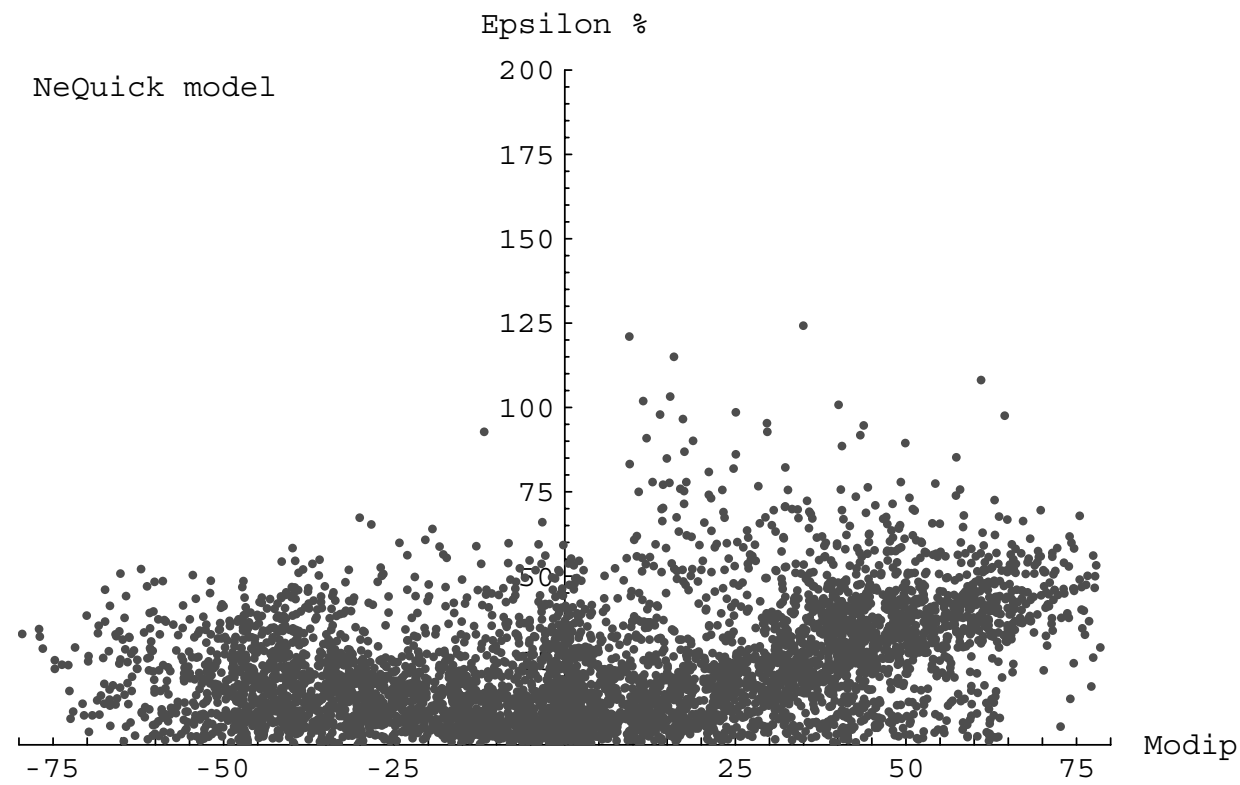

Fig. 3. Scatter plot of $\varepsilon$ computed for IRI model profiles as function of Modified Dip Latitude. 
To have a single number to measure the deviation of the model from the observed profile, we made use of the concept of Epsilon of electron concentration as an objective technique for profile comparisons. $\varepsilon$ (Zhang et al., 1998) is a percentage value defined as the integral from the height $h_{\min }$ to the height $h_{\max }$ of the absolute value of the difference between the modeled and measured electron concentration, over the integral of the measured electron concentration between the same heights, multiplied by 100 .

$$
\varepsilon=\frac{\int_{h_{\min }}^{h_{\max }} \mid N(h)_{\text {model }}-N(h)_{\text {measured }} d h}{\int_{h_{\min }}^{h_{\max }} N(h)_{\text {measured }} d h} \cdot 100 .
$$

Table I. Statistics of $\varepsilon$ in bands of Modified Dip Latitude, considering: profiles covering a height range of 100-300 km above $h_{m} F_{2}$, profiles covering a height range of 400-600 km above $h_{m} F_{2}$, all profiles.

\begin{tabular}{|c|c|c|c|c|c|c|c|}
\hline \multirow{2}{*}{$\begin{array}{l}\text { MoDip } \\
\text { band }\end{array}$} & & \multicolumn{2}{|c|}{$100-300 \mathrm{~km}$} & \multicolumn{2}{|c|}{$400-600 \mathrm{~km}$} & \multicolumn{2}{|c|}{ All ranges } \\
\hline & & NeQuick & IRI & NeQuick & IRI & NeQuick & IRI \\
\hline \multirow{5}{*}{$-90^{\circ}-60^{\circ}$} & No. samples & \multicolumn{2}{|c|}{26} & \multicolumn{2}{|c|}{39} & \multicolumn{2}{|c|}{97} \\
\hline & Mean & 25.0 & 25.2 & 22.1 & 33.4 & 22.1 & 28.7 \\
\hline & St. Dev. & 11.3 & 17.4 & 12.4 & 34.2 & 12.3 & 28.0 \\
\hline & Max & 52.1 & 69.7 & 50.7 & 117.8 & 52.1 & 117.8 \\
\hline & RMS & 27.3 & 30.4 & 25.3 & 47.5 & 25.2 & 40.0 \\
\hline \multirow{5}{*}{$-60^{\circ}-30^{\circ}$} & No. samples & \multicolumn{2}{|c|}{253} & \multicolumn{2}{|c|}{405} & \multicolumn{2}{|c|}{946} \\
\hline & Mean & 19.5 & 27.5 & 18.5 & 19.1 & 18.7 & 21.1 \\
\hline & St. Dev. & 13.1 & 29.8 & 11.5 & 23.5 & 12.0 & 24.9 \\
\hline & $\operatorname{Max}$ & 67.3 & 176.8 & 53.7 & 174.0 & 67.3 & 176.8 \\
\hline & RMS & 23.5 & 40.5 & 21.8 & 30.3 & 22.2 & 32.6 \\
\hline \multirow{5}{*}{$-30^{\circ} 0^{\circ}$} & No. samples & \multicolumn{2}{|c|}{384} & \multicolumn{2}{|c|}{473} & \multicolumn{2}{|c|}{1210} \\
\hline & Mean & 17.9 & 20.5 & 15.2 & 18.4 & 16.0 & 19.4 \\
\hline & St. Dev. & 12.8 & 14.7 & 11.7 & 12.4 & 12.4 & 13.1 \\
\hline & Max & 66.0 & 120.8 & 64.0 & 108.1 & 92.7 & 120.8 \\
\hline & RMS & 22.0 & 25.2 & 19.1 & 22.2 & 20.2 & 23.4 \\
\hline \multirow{5}{*}{$0^{\circ} 30^{\circ}$} & No. samples & \multicolumn{2}{|c|}{411} & \multicolumn{2}{|c|}{445} & \multicolumn{2}{|c|}{1220} \\
\hline & Mean & 19.9 & 18.0 & 20.0 & 17.9 & 19.8 & 18.3 \\
\hline & St. Dev. & 15.7 & 10.6 & 18.4 & 8.8 & 17.5 & 10.0 \\
\hline & Max & 95.3 & 58.7 & 115.0 & 49.1 & 120.9 & 60.6 \\
\hline & RMS & 25.5 & 20.9 & 27.1 & 20.0 & 26.4 & 20.9 \\
\hline \multirow{5}{*}{$30^{\circ} 60^{\circ}$} & No. samples & \multicolumn{2}{|c|}{320} & \multicolumn{2}{|c|}{474} & \multicolumn{2}{|c|}{1263} \\
\hline & Mean & 31.1 & 17.8 & 31.1 & 23.2 & 31.4 & 22.6 \\
\hline & St. Dev. & 14.3 & 15.2 & 15.7 & 21.3 & 16.0 & 20.4 \\
\hline & Max & 85.2 & 121.9 & 88.6 & 138.5 & 124.2 & 138.5 \\
\hline & RMS & 34.2 & 23.4 & 34.8 & 31.5 & 35.2 & 30.4 \\
\hline \multirow{5}{*}{$60^{\circ} 90^{\circ}$} & No. samples & \multicolumn{2}{|c|}{73} & \multicolumn{2}{|c|}{81} & \multicolumn{2}{|c|}{256} \\
\hline & Mean & 47.6 & 51.7 & 38.4 & 54.2 & 40.8 & 52.5 \\
\hline & St. Dev. & 14.4 & 37.9 & 14.5 & 29.3 & 14.9 & 33.7 \\
\hline & Max & 108.1 & 197.0 & 72.5 & 145.2 & 108.1 & 197.0 \\
\hline & RMS & 49.7 & 63.9 & 41.1 & 61.5 & 43.4 & 62.3 \\
\hline
\end{tabular}




\section{Results}

The IZMIRAN database of Intercosmos-19 profiles over a period of two years gives a global coverage of the whole Earth. This means that it is not possible to have instantaneous global maps of electron concentration profiles, but only sets of sparse points, or quasi-longitudinal sections along the satellite path.

NeQuick and IRI topside profiles were computed for the conditions of each measured profile. All the $\varepsilon$ were calculated and the results were statistically evaluated. In particular, it is interesting to examine the different behavior of the models as a function of position. Examining the plots of the computed $\varepsilon$ for each model as a function of the modified dip-latitude (figs. 2 and 3), in the case of IRI it is clear that starting from more or less $30^{\circ}$ the model shows a tendency to diverge from the experimental profiles and this trend increases with increasing modiplatitude. Many cases show an $\varepsilon$ greater than 100 , up to nearly 200 . In the case of the NeQuick model, the situation is very different, it shows a homogeneous behavior at all latitudes, even if the spreading is larger in the Northern Hemisphere. Only few cases have $\varepsilon$ greater than 100 .
Other statistical calculations were computed with these data, like those shown in table I where the results in bands of modified dip-latitude are presented. The table contains statistics of $\varepsilon$ for profiles covering a fixed range of lengths (profiles covering at least $100-300 \mathrm{~km}$ and $400-600 \mathrm{~km}$ above the $F_{2}$ peak) and for all the profiles independently of the range of integration covered by the topside sounding. For each modified diplatitude band the number of samples, the mean value, the standard deviation, the maximum value and the Root Mean Squared of $\varepsilon$ are shown. It appears that the different height ranges of the experimental profiles do not much affect the statistics of $\varepsilon$, which is a quantity dominated by the behavior of the models with respect to experimental data near the $F_{2}$ peak. It can be noticed that while for the NeQuick model there is a general tendency to a better agreement on longer profiles, the IRI shows many cases in which increased height ranges produce greater $\varepsilon$. For the Northern Hemisphere, at high modiplatitudes, the NeQuick model is closer to the experimental data than the IRI model, but at medium and low modiplatitudes the IRI model has a better behavior. For the Southern Hemisphere, the situation is slightly different and the NeQuick model seems to remain closer to

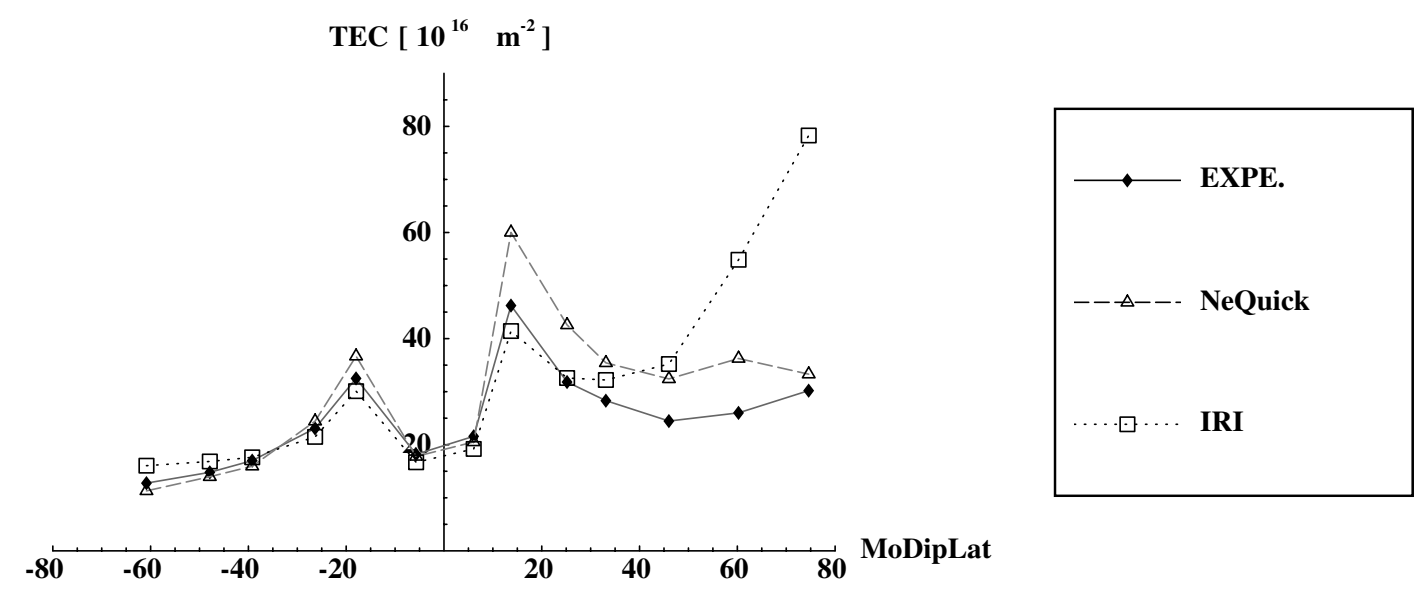

Fig. 4. Cross section of TEC from $F_{2}$ peak to satellite height. 
the experimental profiles: the mean value, the standard deviation, the maximum value and the RMS are always lower than those for the IRI model.

Another kind of analysis that can be done with satellite data is to compare the behavior of the models in predicting the electron content in the available height range of the profile. As an example, fig. 4 presents a quasi-longitudinal (from 204 to $254 \mathrm{E}$ ) section of TEC from peak height to satellite height during a satellite orbit on 28 November 1979 at around noon local time.

At very low Modified Dip Latitude the agreement of both models with experimental data is good, but at high northern latitudes the two models behave in a very different way: the NeQuick slightly overestimates the TEC by a more or less constant value at all modiplatitudes, but the IRI model diverges as the Modified Dip Latitude increases, showing much higher values of TEC than the experimental data at high modiplatitude. This behavior cannot be confirmed for the Southern Hemisphere because of the limited extension of the experimental profiles, near satellite perigee.

\section{Conclusions}

In the present work, the IZMIRAN database of topside electron concentration profile was used to compare the prediction of the IRI and NeQuick models. More than 5000 profiles were used for comparison with each model, in many different geophysical situations, in a period of high solar activity. The concept of $\varepsilon$ was used to give a single number to evaluate the divergence between the experimental and modeled profiles. The $\varepsilon$ were computed for each profile. The two models have shown a similar global behavior, but different specific problems. Cases were found in which there are large discrepancies, particularly in the case of the IRI model at high latitudes. This means that topside modeling is still critical and both models have to be improved in that part of the profile. For the case of the NeQuick model, an improvement is being developed, modifying the empirically determined parameter governing the shape of the topside profile as contained in the present formulation of the model (Radicella and Zhang, 1995).

\section{REFERENCES}

BILITZA, D. (1990): International Reference Ionosphere 1990 NSSDC 90-22, Greenbelt, Maryland.

BILITZA, D. (1994): Topside models: status and future improvements, Adv. Space Res., 14 (12), 17-26.

Di GIOVANNI, G. and S.M. RADICELLA (1990): An analytical model of the electron density profile in the ionosphere, Adv. Space Res., 10, 27.

Hochegger, G., B. Nava, S.M. Radicella and R. LEITINGER (2000): A family of ionospheric models for different uses, Phys. Chem. Earth, 25 (4), 307-310.

RADICELLA, S.M. and R. LEITINGER (2001): The evolution of the DGR approach to model electron density profiles, Adv. Space Res., 27 (1), 35-40.

RADICELLA, S.M. and M.-L. ZHANG (1995): The improved DGR analytical model of electron density height profile and total electron content in the ionosphere, Ann. Geofis., 38 (1), 35-41.

ZHANG, M.-L., S.M. RADICELLA and L. KERSLEY (1998): Measured topside electron profile and its modeling, in Proceedings of the IRI Task Force Activity 1997, IC/IR/ 98/9 Trieste, June 1998. 